\title{
Normal Leucocytes, as They Appear under the Copper Peroxidase Reaction.
}

50th* Report of the Peroxidase Reaction.

\author{
By
}

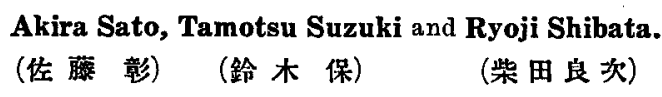

(From the Pediatric Department, Faculty of Medicine, Tohoku Imperial University, Sendai. Director: Prof. A. Sato.)

Since the device of the Copper Peroxidase Reaction by A. Sato, ${ }^{1)}$ one of the authors, and S. Sekiy ${ }^{1)}$ in 1919 , some modifications of the method have been tried by different authors. They will be related in full details in a later report. Here in the present treatise only the original copper peroxidase method of Sato and Sekiy a, and the Tohoku-Pediatric Method, a modification of the above method, will be considered.

\section{Sato-Sekiya's Method.}

I. Reagents Used.

A. Solution A. Copper sulphate solution, 0.5 per cent.

B. Solution B. Preparation: Rub $0.2 \mathrm{gm}$. of benzidine with a few drops of water in a mortar. Add 200 c.c. of water at room tem-

* Availing myself of the publication of the 50th Report of Peroxidase Reaction, I should like to show here where the papers from the 1 st to the 4 th, from the 8 th to the 9 th and the 17 th paper were published.

The 1st Report of the Peroxidase Reaction, A.S to and Sh. Yoshimats $\mathrm{n}$, The Peroxidase Reaction in Epidemic Encephalitis, Am, J. Dis. Child., 1925, 29, 301.

The 2nd Report., A. S a to, The Localisation of a Lesion in the Brain by Differential Staining of Blood Smears, Am. J. Dis. Child., 1925, 29, 313.

The 3rd Report., A. S a to and S e k i a, A Simple Method for Differentiation of Myeloic and Lymphatic Leucocytes of the Human Blood, Tohoku J. Exp. Med. 1926, 7, 111.

The 4th Report., S h. Y o s hi mats u, Experimentation on "Peroxidase-Punctured" Animals, Tohoku J. Exp. Med., 1926, 7, 116.

The 8th Report., A. Sato, Two Methods for the Eosinophile Count in the Counting Chamber for Routine Work, Journ. Laboratory and Clinical Med., 1928, 13, 1056.

The 9th Report, A.S a to and K.Sh oji, Counting Chamber Peroxidase Method for Blood. Simultaneous Rapid Differential Leucocyte Count and Total Leucocy te Count, Journ. Laboratory and Clinical Med., 1928, 13, 1058.

The 17th Report., not yet published.

Prof. A. Sato.

1) A. Sato and S. Sekiy a, Tohoku Igaku-Zasshi, 1920, 6, 534; Tohoku J. Exp. Med., 1926, 7, 111. 
perature and filter. To the filtrate add 4 drops of hydrogen peroxide (3 per cent.). If kept in a brown bottle in the dark when not in use, this preparation will last a year, although precipitation will begin to occur soon after preparation.

C. Solution C (counter stain). Safranin, aqueous solution, 1 per cent.

II. Technique.

To a fresh, dry blood smear apply Solution A. Pour off most of it and apply Solution B for two minutes. Then wash thoroughly with water, and add Solution $\mathrm{C}$ at once and let stand for two minutes before washing.

\section{The Tohoku-Pediatric Method.}

K. Suzuki ${ }^{2)}$ modified the original Sato-Sekiya's peroxidase stain for the purpose of investigating the nuclear shift (Sato-Sekiya-Suzuki's method). Now this modification had undergone some modifications again in the hematological laboratory of our TohokuPediatric Department, so that this new modification may as well be called the Tohoku-Pediatric Method* (for Human Blood).

I. Reagents Used.

A. Solution A. Copper sulphate solution, 10 per cent.

B. Solution B. Saturated solution of benzidine, to which $3 \%$ hydrogen peroxide was added in the amount of 2 drops per 100 c.c.

C. Solution C. Safranin, aqueous solution, 1 per cent.

II. Technique.

Pour Solution A half full on a fresh, air-dried blood smear, and then few drops of Solution $C$ and mix. After a lapse of four or five minutes pour off most of them and apply Solution B on the smear and wait about one minute. Wash thoroughly with running water and then dry.

Main Differences of Blood Films between the Two Stain Methods.

What is a striking difference between blood films treated with these methods? The main differences will be related below:-

2) K. S u u k i, Tohoku J. Exp. Med., 1929, 12, 224.

* As to the method for rabbit blood it will be related in a later report, together with modifications of the $\mathrm{S}$ a to-Sekiy a Peroxidase Reaction. 
1. A fresh blood film treated with the original Sato-Sekiya's method will retain red cells as well as leucocytes, while another treated with the Tohoku-Pediatric Method will retain only white cells. At first thought the latter method may be considered as inferior to the former in this respect. But in daily hematological work blood films with leucocytes alone are often more convenient than ones with red cells retained. Besides, erythroblasts are kept remaining on the film; these belong of course to the category of red cells.

2. With the weak power objective myeloid leucocytes treated with Sato-Sekiya's method appear generally far darker blue than those treated with Tohoku-Pediatric method; that is, with the highpower objective, the former shows much darker and larger blue-stained peroxidase-granules than the latter.

Taking into consideration the fact that in Gies ma-stained blood films a differentiation between some cells of the myeloid system and of the lymphatic is often difficult, the former-Sato-Sekiya's method-may appear far better than the latter method, because the peroxidase reaction-which is characteristic of the myeloid system-will appear much more intensive with the former method. But here again the latter (Tohoku-Pediatric) Method is preferable to the former in some instances; for example, it is much easier to count nuclear lobulation on the blood film treated with the latter. method than with Sato-Sekiya's method (Cf. Fig. 1).

\section{Differences of Normal Leucocytes between the \\ Two Stain Mcthods.}

\section{A. Neutrophiles (Cf. Figss. 1, 2, 3 and 4).}

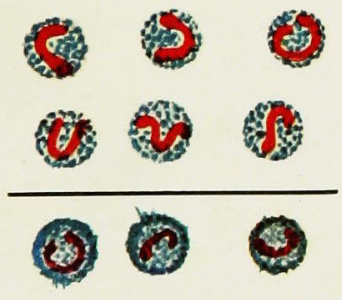

Fig. 1. Neutrophiles ("Stabkernig"). Above: Tohoku-Pediatric Method Below: Sato-Sekiya's original stain

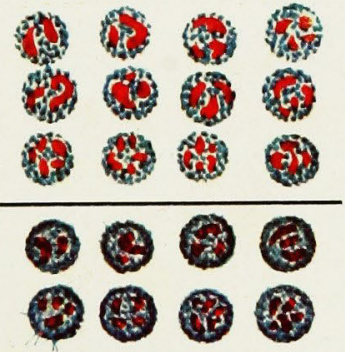

Fig. 2. Nentrophiles ("Segmentkernig"). Above: Tohoku-Pediatric Method Below: Sato-Sekiya's original stain 


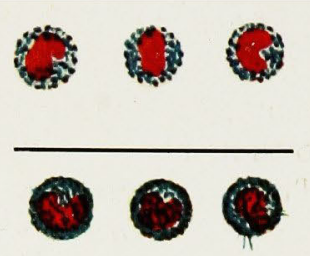

Fig. 3. Neutrophiles ("Young forms"). Above: Tohoku-Pediatric Method

Below: Sato-Sekiya's original stain

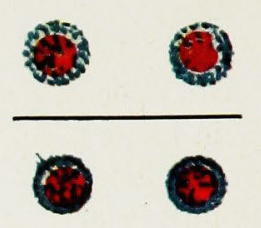

Fig. 4. Neutrophiles ("Myelocytes"). Above: Tohoku-Pediatric Method

Below: S a to-Sekiy a's original stain

1. Under the original stain of Sa to-Sekiy a neutrophiles are so dark blue that they are very easily distinguishable from those of monocytes, while they are not so dark in colour as the granules of eosinophiles. This differentiation is again very easy, though it will be related later. While the peroxidase granules in neutrophiles are thus dark stained and show without the slightest doubt that they are myeloid elements, they make the outline of even safranin-stained nuclei of neutrophiles less distinct, so that the method is inferior to the Tohoku-Pediatric method when it is matter concerning Arneth-Schilling's nuclear shift, for instance (Cf. Figs. 1, 2, 3 and 4).

2. Under the Tohoku-Pediatric method with safranin counter stain, peroxidase granules in neutrophiles are lighter in colour than under Sato-Sekiya's stain, but distinct enough to show that the cell in question is a neutrophile and to enable one to-so to speak - count the granules one by one. Here safranin-stained nuclei are very conspicuous, so that their features are very clear-cut.

B. Eosinephiles (Cf. Fig. 5).

1. Under the Sato-Sekiya's method, peroxidase in eosinophiles are stained as very, very dark blue coarse granules, so that one blue stained eosinophile leucocyte among several similarly
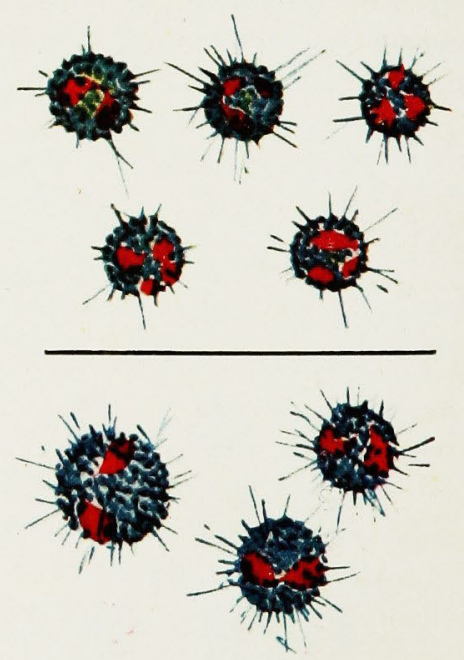

Fig. 5. Eosinophiles.

Above: Tohoku-Pediatric Method

Below: Sato-Sekiya's original stain 
blue-stained neutrophiles will be easily recognized at one glance even by a beginner. Besides, it is very characteristic of eosinophiles, that blue needle-shaped crystals (or crystal-like things) protrude from cell bodies or lie formed very close to them, so that the crystals appear like fireworks photographed (Cf. Fig. 5). These crystals may be considered as purely artificial products which would not occur with due technique. But the fact is different; it has much to do with peroxidase itself or peroxidase granules and must have some biological significance or other. So, S. Konn $0^{\text {i) }}$ of the Laryngo-Rhino-Otological Clinic in our University, who made an investigation into the copper peroxidase reaction of the pus in acute and chronic otitics cases attributed some prognostic value to the crystals. A definite significance may be attributed to the crystal-like products ${ }^{4}$ in the future. It should be borne in mind that the needle-like crystals can be formed in the case of neutrophiles too and also in some monocytes, especially when the peroxidase stain is applied for a longer time.

2. Peroxidase granules in eosinophiles are, even under the Tohoku-Pediatric method, stained very dark with not a few crystal-like products, as in the case of the original Sato-Sekiy a stain. Central parts of the cytoplasma are often stained light brown due to safranin used as counter stain.

The outlines of nuclei in eosinophiles are more easily recognized by this method than by the original Sa to-Sekiya, though the difference is not, due to their particular form, so striking as in the case of neutrophiles.

\section{Basophiles (Cf. Fig. 6).}

As to basophiles, there is no special difference between the two peroxidase stain methods, because they are, as already shown by K. Tok u é, ${ }^{5}$ almost peroxidase-negative (Cf. his report). As to the question whether basophiles are peroxidase-positive or negative, Tok u $\mathrm{e}^{4}$ ) has already reported full de-

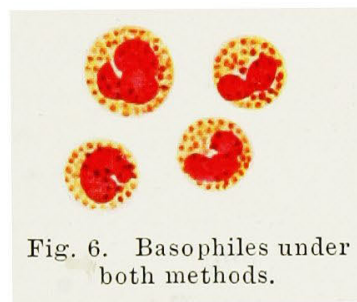
tails concerning the problem, but a further treatise bearing on the point will be reported from our Laboratory.

As will be shown in Fig. 6, they will appear in the absence of leucocytes of the other kinds as if they were peroxidase-posive, be-

3) S. Ko n n o, Tohoku J. Exp. Med., 1931, 17, 31.

4) As to K. Lö wy's opinion concerning the crystal like formations in SatoSekiya's method, we expect to relate about it in a paper in which different modifications of the method are to be discussed (Wien. med. Wschr., 1931, 81, 1586).

5) K. Tok ué, Tohoku J. Exp. Med., 1929, 12, 459. 
cause they may appear l1ke eosinophiles stained with benzidine-peroxidase stain without the use of copper solution, and the peroxidasenegative basophile granules are brownish stained with safranin solution alone. It should be noted here that the granules of eosinophiles are stained likewise if stained with safranin alone. Of course no mistake can arise, when a blood film is examined, as to the negativity of the peroxidase reaction, because these brown granuled basophiles are seen, if at all, only scattered among blue-stained peroxidase-positive cells.

As to the brown colour of basophile granules, we shall tell about it later (Cf. Plasma cells).

\section{Monocytes (Cf. Fig. 7).}

1. Under the original SatoSekiya stain, granules of monocytes including transition forms are stained blue in faintly safranin-red stained cytoplasma. $\mathrm{Nu}$ clei are certainly stained red with safranin, but considerably more lightly than those of neutrophiles and of lymphocytes. In a word, monocytes do not appear so dark as neutrophiles, and their nuclei do not appear so dark red as those of neutrophiles.

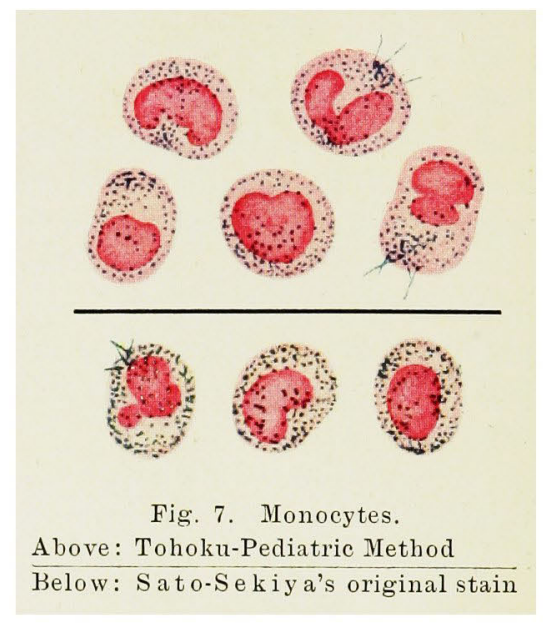

2. With the Tohoku-Pediatric method monocytes appear lighter blue with slightly darker red cytoplasma and nuclei. Outlines of nuclei appear sharper than under the original Sato-Sekiy a stain, and it is characteristic of monocytes that blue granules appear densely grouped in one corner. These characteristics are seen more distinctly under the present method than under the original Sato-Sekiya stain.

Crystal-like formations, as described in eosinophiles, appear in some cells under both methods, though much less remarkably (Cf. Fig. 7).

E. Lymphocytes (Cf. Fig. 8).

They appear light-red in cytoplasma and dark-red in nucleus under

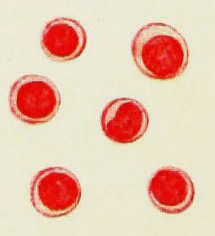

Fig. 8. Lymphocytes, under the Tohoku-Pediatric Method as well as under the original S a toSekiy a stain, 
the Tohoku-Pediatric Method as well as under the original SatoSekiya.

\section{Remarks.}

As for abnormal and pathological leucocytes, as well as plasma cells including Türk's form, we shall make a treatise on them in a later report.

\section{Summary.}

In the present paper it has been discussed how normal leucocytes, peroxidase-negative as well as peroxidase-positive, will appear under the original copper peroxidase reaction of Sato-Sekiya and under the Tohoku-Pediatric Method, a modification of the original. And it has been shown how easily the leucocytes can be differentiated with these two methods, especially with the Tohoku-Pediatric Method. 